\title{
State of the art und Zukunft der Vitrektomie - Techniken und Instrumente
}

\author{
Expertenmeinung und Literaturübersicht
}

\author{
Stefan Mennel (D) - Nikolaos E. Bechrakis · Susanne Binder · Anton Haas
}

Eingegangen: 17. Juli 2017 / Angenommen: 29. Juli 2017 / Online publiziert: 5. September 2017

(C) The Author(s) 2017. Dieser Artikel ist eine Open-Access-Publikation.

\begin{abstract}
Zusammenfassung
Hintergrund Durch die Einführung der Pars plana Vitrektomie ist eine Vielzahl von vitreoretinalen Erkrankungen behandelbar geworden. Kontinuierliche Verbesserungen der Geräte, Instrumente und zusätzliche Hilfsmittel sowie neue Techniken kennzeichnen den enormen Fortschritt der vitreoretinalen Chirurgie.

Methoden In einer Literaturübersicht werden die Operationstechniken verschiedenster vitreoretinaler Eingriffe analysiert. Sofern aufgrund aktueller Entwicklungen verschiedene Optionen in der Operationstechnik bestehen, werden Experten auf dem Gebiet der vitreoretinalen Chirurgie über ihre Erfahrung und ihre Empfehlung befragt.

Ergebnisse Die 20 Gauge Pars plana Vitrektomie wurde größtenteils durch die Verwendung von Trokaren (23, 25 und 27 Gauge) mit entsprechenden Instrumenten mit kleinerem Durchmesser abgelöst. Die Pars plana Vitrektomie ist nahtlos möglich, trotzdem kann es notwendig sein, eine zusätzliche Sicherung der Wunde mit einer Naht durchzuführen, um Leckage und Hypotonie zu vermeiden. Die Visualisierung des Glaskörpers erfolgt zunehmend mit Triamcino-
\end{abstract}

Prof. Dr. S. Mennel, FEBO (夰)

Augenabteilung, LKH-Feldkirch,

Carinagasse 47, 6800 Feldkirch, Österreich

stefan.mennel@gmail.com

Prof. Dr. S. Mennel, FEBO

Philipps Universität Marburg, Marburg, Deutschland

Prof. Dr. Dr.h.c. N. E. Bechrakis, FEBO

Universitätsklinik für Augenheilkunde und Optometrie,

Medizinische Universität Innsbruck, Innsbruck, Österreich

Prof. Dr. S. Binder

Sigmund Freud Privatuniversität Wien, Wien, Österreich

Prof. Dr. A. Haas

Augenklinik, Universität Graz, Graz, Österreich lon, epiretinale Gliosen und die Membrana limitans interna werden routinemäßig mit Vitalfarbstoffen dargestellt. Bei der Operationstechnik beim Makulaforamen und bei der Ablatio retinae zeigen sich sowohl bei aktuellen Publikationen als auch unter den Experten Variationen.

Schlussfolgerungen Die Pars plana Vitrektomie hat sich aufgrund der Weiterentwicklung speziell im Bereich der Trokar Systeme, Vitrektome, der WeitwinkelBeobachtungssysteme sowie dank neuer Techniken zu einer essentiellen OP-Methode für ein weites Indikationsspektrum entwickelt. Dies ist die Basis der Behandlung verschiedenster vitreoretinaler Erkrankungen mit immer weniger Operationstrauma, weniger intraoperativen und postoperativen Komplikationen und gutem Therapieerfolg. Unterschiedliche Operationsvarianten, neue Techniken und die Weiterentwicklung der Geräte und Instrumente sind die Basis für einen auch zukünftigen Fortschritt der Pars plana Vitrektomie.

Schlüsselwörter Pars plana Vitrektomie - Makulaforamen - Ablatio retinae - Operationstechnik · Endoillumination

\section{State of the art and future of vitrectomy - techniques and instruments \\ Expert opinion and review of the literature}

\section{Summary}

Background Due to the introduction of pars plana vitrectomy numerous vitreoretinal pathologies became effectively treatable. Continuous improvement of vitrectomy devices, instruments and additional adjuvants as well as new techniques display the progress in vitreoretinal surgery.

Methods In a literature review current techniques of vitrectomy in several vitreoretinal pathologies are 
analysed. Concerning new developments of surgical techniques, experts on the field were interviewed for their experience and recommendations.

Results The classic 20 gauge pars plana vitrecomy has been modified by new small trocar systems $(23,25$ and 27 gauge) and corresponding instruments with smaller diameters. In pars plana vitrectomy a sutureless procedure is possible but for security reasons a suture of the wound may be necessary to prevent leackage and hypotony. Visualization of the vitreous is principally ensured by triamcinolone. Staining of epiretinal membranes and of the internal limiting membrane with vital dyes is well established today. However, surgical techniques in the treatment of macular holes and of retinal detachment vary in the current literature and expert opinion.

Conclusions Due to past and current developments of pars plana vitrectomy especially in regard of trocar systems, vitreous cutters and wide-angle viewing systems as well as the establishment of new techniques a widespread acceptance for the treatment of numerous vitreoretinal pathologies has emerged.

This progress in the treatment of vitreoretinal diseases enables less operative trauma, reduced intraoperative and postoperative complications and enhanced final success. Variable operation procedures, new techniques and development of new devices and instruments are essential for further progress in the future of pars plana vitrectomy.

Keywords Vitrectomy $\cdot$ Macular hole $\cdot$ Retinal detachment $\cdot$ New instruments $\cdot$ Endoillumination

Die Vitrektomie wurde im Jahre 1970 durch Machemer et al. [1, 2] eingeführt. Vor dieser Zeit wurde Glaskörper durch die „Open-sky-Öffnung“ mittels Pinzette und Schere oder mittels Tupfer und Schere (Tupfervitrektomie) entfernt [3]. Einerseits wurde mit diesen zwei Techniken vorgefallener Glaskörper entfernt, andererseits musste zur gezielten Glaskörperentfernung die Hornhaut eröffnet und die Linse entfernt werden [3]. Mit der Entwicklung eines Saug-Schneidegerätes und durch das Arbeiten in einem geschlossenen System, um eine Druckabfall während der OP zu verhindern, sowie durch den Zugang über die Pars plana, wurden essentielle Schritte gesetzt [3, 4]. Die ersten Vitrektome hatten einen Durchmesser von 17 Gauge. Mit der Einführung der 20 Gauge 3-Port Pars plana Vitrekomie (ppV) wurde die Voraussetzung geschaffen, diese Operationstechnik für verschiedenste Indikationen vitreoretinaler Erkrankungen nützen zu können. Vorerst nur für Glaskörpertrübungen und Blutungen verwendet, hat sich im Verlauf das Indikationsgebiet der ppV auf Netzhautablösungen, proliferative Vitreoretinopathien, vitreoretinale Traktionen, diabetische Retinopathien, Macular Pucker, Makulaforamen, subretinale chirurgische Eingriffe und Tumorentfernungen ausgeweitet. Durch die relativ sichere Anwendung werden heute von einigen Chirurgen auch Glaskörpertrübungen im Sinne von Mouches volantes als Indikationsspektrum der ppV gesehen. Um dies alles zu ermöglichen, wurden enorme Entwicklungen beim Vitrektom, der Qualität und Größe der Instrumente, der Endoillumination und Endolaserkoagulation, der Visualisierung des Glaskörpers und des Fundus mit Kontaktglas und der kontaktlosen Weitwinkel Beobachtung gemacht [5]. Die Verwendung von Luft, spezifischer Gase, schwerer Flüssigkeiten und verschiedenster Silikonöle hat wesentlich dazu beigetragen, die Erfolgsrate bei abgehobener Netzhaut zu verbessern [6].

Trotz der enormen Fortschritte der vitreoretinalen Chirurgie sind die Entwicklungen nicht abgeschlossen und auch die von den vitreoretinalen Chirurgen verwendeten Techniken sind in Abhängigkeit der Erkrankung variabel. Dies führt dazu, dass aktuell verschiedenste Instrumentengrößen in Verwendung sind und auch große Variationen bestehen wie z. Bsp.: bei der Verwendung von Endotamponaden, Anwendung von Vitalfarbstoffen zur Visualisierung von transparenten Strukturen, Indikationsstellung zur Durchführung einer kompletten ppV oder einer Core-Vitrektomie bei Erkrankungen der Makula sowie bei der Operationstechnik von Makulaforamen und im Speziellen bei der Re-Operation persistierender Makulaformen. Auch bei der Behandlung der Netzhautablösung sind unterschiedliche Techniken möglich. Die Operation der Ablatio retinae kann als Plombenoperation und somit als rein extraokulärer Eingriff erfolgen. Allerdings hat die ppV enorme Fortschritte gemacht, sodass sie von vielen Experten bevorzugt wird. Ist die Plombenoperation als rein extraokulärer Eingriff nicht mehr so populär weil die ppV enorme Fortschritte gemacht hat? Ist die zusätzliche Cerclage bei der Vitrektomie heute noch notwendig? In diesem Zusammenhang stellt sich die Frage nach dem „state of the art“ der Vitrektomie aktuell und welche zukünftigen Entwicklungen sich abzeichnen. In dieser Übersichtsarbeit sollen mögliche Variationen bei der Verwendung von Instrumenten und Geräten, Flüssigkeiten und Farbstoffen sowie die Anwendung unterschiedlicher Techniken anhand der aktuellen Literatur dargestellt und dann die Meinung von ausgewiesen Experten der vitreoretinalen Chirurgie eingeholt werden.

\section{Sklerotomien und Größe der Instrumente}

Die 20 Gauge 3-Port ppV war über nahezu vier Jahrzehnte der technische Standard der Vitrektomie. Die skleralen Zugänge und die Bindehautöffnung mussten durch Nähte verschlossen werden. Vergleichbar zur nahtlosen Kataraktchirurgie wurden im weiteren Verlauf tunnelförmige Sklerazugänge entwickelt, um auch die Vitrektomie nahtlos durchführen zu können [7, 8]. Trotz dieser Möglichkeit haben die meisten vitreoretinalen Chirurgen weiterhin den Nahtverschluss der Sklera- und Bindehautöffnung bevorzugt. Da 
Komplikationen im Bereich von Sklerotomien gehäuft auftreten, wurden Versuche unternommen, die Anzahl der Sklerotomien zu reduzieren [9]. Im Speziellen kann bei vorbestehender Pseudophakie die Infusion mittels Parazentese über einen Vorderkammerzugang erfolgen [10]. Erst durch die Entwicklung von Trokaren und Instrumenten mit einem kleineren Durchmesser konnte sich die nahtlose Vitrektomie etablieren. Es ist dabei zu berücksichtigen, dass die 20 Gauge ppV keine Trokare verwendet und sich somit die 20 Gauge auf die Größe der Sklerotomie bezieht. Bei der small Gauge ppV mit Trokaren entsprechen die Gauge einem Maß für den Durchmesser der Instrumente, aber nicht für jenen des Trokars, der durch die Binderhaut und Sklera geführt wird. Im Jahr 2002 wurde erstmals die nahtlose 25 Gauge ppV vorgestellt [11]. Für die klinische Anwendung noch nicht optimale FlowParameter und nicht ausreichend stabile Instrumente waren der Grund, dass 2005 die 23 Gauge nahtlose ppV mit etwas größerem Durchmesser von Trokaren und Instrumenten präsentiert wurde und sich diese Methode parallel dazu etabliert hat [12]. Im Verlauf wurden die Flow-Parameter und die Instrumente derart verbessert, dass 2010 bereits die 27 Gauge nahtlose $\mathrm{ppV}$ vorgestellt wurde [13]. Im postoperativen Verlauf einer ppV können Hypotonien auftreten, weshalb einige Chirurgen nach Entfernung der Trokare die Zugänge mittels einer Naht im Wundbereich sichern. Die Bezeichnung 23-, 25- oder 27 Gauge ppV ist somit nicht mit einer nahtlosen Vitrektomie automatisch gleichzusetzen.

\section{Fragen}

Welches Gauge System verwenden Sie hauptsächlich und sollten Sie zusätzliche verwenden, dann aus welchem Grund? In welchen Situationen nähen Sie die Sklerotomie nach Entfernung der Trokare? Führen Sie zum besseren Wundverschluss eine Lufttamponade durch?

Verwenden Sie auch Trokare bei Re-Operationen, wenn die primäre Operation nicht von Ihnen durchgeführt wurde oder als 20 Gauge ppV erfolgte? Wie sehen Sie die zukünftige Entwicklung?

\section{Nikolaos Bechrakis}

Für die Makulachirurgie verwende ich als Standard die 27 Gauge, ansonsten (Ablatio, PDR) die 25 Gauge Zugänge. Bei Ausnahmefällen (z. B. schweres Trauma) kommen auch selten die 20 Gauge Instrumente zur Anwendung. Nach Entfernung der Trokare nähe ich die Sklerotomien bei Undichtigkeit.

Eine Lufttamponde zum besseren Wundverschluss verwende ich bei der $27 \mathrm{G}$ Vitrektomie nicht, außer die Netzhaut benötigt eine Tamponade.

Trokare verwende ich auch bei Re-OP's, manchmal auch nach Eröffnung der Bindehaut.
In Zukunft wird sich die kleinlumige ( $23 \mathrm{~g} / 25 \mathrm{~g} / 27 \mathrm{~g})$ trokargeführte Vitrektomie weiter festigen und in der Anwenderfreundlichkeit verbessern.

\section{Susanne Binder}

Ich verwende 23 Gauge für alle komplexeren Fälle und nähe die Sklerotomien immer zu, wenn ich Silikonöl als Tamponade einsetze.

Für einfache Fälle, wie etwa ein Membranpeeling, benutze ich in zunehmender Frequenz 27 Gauge Instrumente, da durch die sehr kleine Wundöffnung rasch ein sicherer Wundverschluss entsteht. 25 Gauge ist eine gute Mittelgröße, die man zur Zeit sowohl für schwierige als auch für leichte Fälle einsetzen kann, und müsste ich mich für nur eine Größe entscheiden, dann wäre es 25 Gauge.

Eine Lufttamponade führe ich immer durch, auch um postoperative Hypotonien zu vermeiden.

Ich verwende immer Trokare, die ich nur dann entferne, wenn ich z. B. eine Sklerotomie vergrößern muss, um einen intraokularen Fremdkörper zu entfernen. Danach verschließe ich aber diese Wunde wieder partiell und setze den Trokar ein, da er den Augendruck stabiler hält. Bei Reoperationen versuche ich - soweit möglich - an einer anderen Stelle einzugehen als bei der vorgehenden Operation. Die Sklera ist dann noch intakt und die Trokare sitzen besser. Darüberhinaus ist die Blutungsneigung geringer, wenn man nicht in ein Narbengebiet sticht.

Die Zukunft des Instrumentendesigns ist nicht abzusehen. Es gibt schon Einzelberichte über 29 Gauge Instrumente. Dennoch glaube ich, dass die Verbesserungsmöglichkeit eher in der Schneid-Saugqualität der Cutterinstrumente und verbesserten Software der Geräte liegen wird, wodurch uns eine noch sicherere und raschere Glaskörperentfernung ermöglicht werden kann.

\section{Anton Haas}

Prinzipiell verwende ich ein 23 Gauge System, da unsere apparative Ausstattung damit am effizientesten arbeitet. In einem anderen Krankenhaus arbeite ich mit einem Gerät der neuen Generation mit der 25 Gauge Vitrektomie und sehe keinen wirklichen Unterschied zu 23 Gauge, sodass ich das 25 Gauge System in Zukunft für die meisten Vitrektomien einsetzen werde. Die Instrumente bei der 27 Gauge Vitrektomie sind merklich biegsamer, und ich würde sie eher nur bei der Makulachirurgie und nicht bei Netzhautablösungen einsetzen.

Sobald ich merke, dass eine Sklerotomie undicht ist, sprich es kommt Flüssigkeit, Gas oder Silikonöl aus der skleralen Inzision heraus, setze ich eine Naht. Gerade bei jungen und myopen PatientInnen ist es fast ein Muss, die 23 Gauge Sklerotomien zu vernähen.

Eine Lufttamponade nur zum besseren Wundverschluss führe ich nicht durch. 
Bei den Reoperationen, egal nach 20 Gauge oder kleiner, verwende ich ein Trokarsystem, nur muss ich hier öfters eine Naht zum Wundverschluss legen.

\section{Core-Vitrektomie}

Die Core-Vitrektomie beschreibt die Entfernung des zentralen Glaskörpers. Bei einer totalen oder peripheren ppV erfolgt eine möglichst komplette Entfernung des Glaskörpers. Speziell bei Pathologien in der Netzhautperipherie ist die penible Vitrektomie in diesem Bereich essentiell. Die Entfernung von vitreoretinalen Traktionen, das Auffinden von Netzhautdefekten, die Entfernung von Membranen bei der proliferativen Vitreoretinopathie sind effektiv, um postoperative Komplikationen zu vermeiden und die Re-Operationsrate zu senken. Die Vitrektomie im Bereich der Netzhautperipherie und der Glaskörperbasis ist trotz der Anwendung der Weitwinkel-Beobachtungsysteme sehr aufwendig und zeitintensiv. Mehrere Methoden stehen für diesen Operationsschritt zur Verfügung. Einerseits kann ein Assistent die Peripherie eindellen und somit dem Chirurgen die peripheren Bereiche in den visualisierbaren Strahlengang bewegen, andererseits kann der Chirurg auch ohne Assistenz die Peripherie mit der Lichtquelle eindellen [14]. Hierbei handelt es sich um eine transsklerale Illumination der Netzhautperipherie. Die Helligkeit ist dadurch geringer, aber ausreichend für die Vitrektomie an der Glaskörperbasis, sofern keine subretinalen Blutungen im betroffenen Areal vorhanden sind. Eine weitere Möglichkeit ist die Verwendung einer Endoillumination über bestehende Zugänge oder über die Verwendung zusätzlicher Zugänge an der Pars plana [15]. In diesem Fall ist es möglich, mit einer Hand die Vitrektomie durchzuführen und mit der anderen Hand selber die Peripherie einzudellen.

\section{Fragen}

Führen Sie eine Core-Vitrektomie durch und bei welchen Indikationen? Welche Vorteile sehen Sie in der Core-Vitrektomie? Welche Technik empfehlen Sie für die periphere Vitrektomie? Welche Beleuchtungsmöglichkeiten werden sich Ihrer Meinung nach etablieren?

\section{Nikolaos Bechrakis}

Ich fange immer mit einer Core-Vitrektomie an und erweitere diese zu einer Vitrektomie bis zur GK-Basis, wenn dies notwendig ist (wenn die Pathologie dort liegt).

\section{Susanne Binder}

Eine Core-Vitrektomie führe ich ganz selten durch, da ich auch bei der Indikation eines Membranpeelings und Makulaforamen den Glaskörper bis nach anterior reinige. Eine mögliche Indikation wären aber Jugendliche mit z. B. traumatischem Makulaforamen oder Optic pit, damit hier eine Kataraktentwicklung möglichst hintangehalten wird.

Ich intendiere entweder selbst oder lasse meine Assistenz intendieren, wenn ich die Glaskörperbasis abtragen möchte. Dies scheint mir genauer als nur die Sicht durch die - zugegebenermaßen jetzt viel besseren - Weitwinkelsysteme.

Chandelierlichter werden nun viel häufiger eingesetzt als früher. Sie helfen enorm, da man dadurch beide Hände für die Präparationsarbeit nahe an der Netzhaut zur Verfügung hat. Manche Kollegen verwenden sogar 2 zusätzliche Chandelierlichter, um das Augeninnere besser auszuleuchten.

Da es früher toxische Lichtschäden besonders bei Manipulationen an der Makula gab, ist man heute vom grellen Xenonlicht wieder abgekommen. Auch benötigen wir Chirurgen jetzt weniger Zeit für das Membranpeeling, was die Lichtbelastung senkt.

\section{Anton Haas}

Ich führe bei allen makulachirurgischen Eingriffen nur eine Core-Vitrektomie durch.

Der Vorteil der Core-Vitrektomie ist die Vermeidung von iatrogenen peripheren Netzhautschäden. Wenn der Glaskörper noch anliegend ist, so hebe ich ihn nur bis zur mittleren Peripherie ab, um periphere Netzhautdefekte $\mathrm{zu}$ vermeiden.

Bei der Chirurgie der Ablatio oder bei diabetischen Indikationen säubere ich die Glaskörperbasis genau. Dabei verwende ich eine transsklerale Illumination oder das Spaltlicht am Mikroskop und delle ein. Da mich das Chandelierlicht blendet und $\mathrm{zu}$ wenig fokussiert ist, benutze ich es nur, wenn die Netzhautsituation ein bimanuelles Arbeiten erfordert.

Inwieweit andere Farbspektren einen Vorteil bringen, werden wir sehen. Sonst glaube ich, dass die bisherige Palette der Beleuchtungsmöglichkeiten ausreicht, um die meisten Indikationen damit $\mathrm{zu}$ operieren. Ändern wird sich nur die Beleuchtungsquelle, wobei derzeit auf LED-Licht gesetzt wird.

\section{Vitreomakuläre Traktion - Behandlungsmethode}

Während vitreomakuläre Traktionen (VMT) früher nur in ausgeprägten Formen durch die Fundusuntersuchung erkennbar waren, ermöglicht die optische Kohärenztomographie (OCT) detailliert die Visualisierung der Glaskörperanheftung an der Oberfläche der Neuroretina. Somit können Fälle von VMT von vitreomakulärer Adhäsion (VMA), bei welcher eine fokale Anheftung ohne morphologische Veränderungen der Neuroretina vorliegt, differenziert werden. Es stellt sich heute vor allem die Frage nach der Indikation einer Intervention und auch die Frage welche Operationsmethode angewandt wird. Neben der chirurgischen Lösung der VMT und der eventuellen Entfernung von epiretinalen Membranen mittels Vitrektomie besteht seit 2012 die Möglichkeit der enzymatischen Vitreolyse [16-19]. Obwohl schon 
vor Jahren die Induktion einer Glaskörperabhebung durch intravitreale Gasapplikation gezeigt wurde, hat diese Methode erst in den letzten Jahren bei der VMT ein vielversprechendes Indikationsgebiet gefunden [20-22].

\section{Fragen}

Welche Voraussetzungen müssen vorliegen, dass Sie bei VMT eine Therapieoption anbieten? Welche Therapie bieten Sie an und ist diese abhängig vom Befund? Wie schätzen Sie die zukünftigen Entwicklungen ein? Führen Sie eine Visualisierung des Glaskörpers im Rahmen der Vitrektomie durch und wie machen Sie dies?

\section{Nikolaos Bechrakis}

Wenn eine Indikation zur Behandlung der VMT vorliegt (hauptsächlich Symptomatik und/oder Visusabfall), biete ich eine Vitrektomie an, sonst biete ich keine alternative Behandlungsoption an. Eine Visualisierung des Glaskörpers mittels Triamcinolon führe ich nur in Ausnahmefällen durch (z. Bsp. vermutete Vitreoschisis bei PDR).

\section{Susanne Binder}

Es muss der Patient Symptome wie Metamorphopsien oder Sehverschlechterung haben und im OCT nicht nur die Glaskörpertraktion erkennbar sein, sondern auch eine beginnende Veränderung in den Netzhautschichten. Solange die Netzhaut völlig intakt ist, beobachte ich. Dies gilt auch für sogenannte „drohende“ oder „imminente“ Makulaforamen, bei denen das Partnerauge schon ein penetrierendes Makulaforamen hatte. Hier zeigt die Literatur, dass es immer wieder zu spontaner Separation der Traktion kommt und empfiehlt engmaschige Kontrollen.

Bei jungen, phaken Patienten mit einer isolierten Traktion, die kleiner als $1,5 \mathrm{~mm}$ ist und keine epiretinalen Membranen haben, biete ich auch Jetrea ${ }^{\circledR}$ (Mikroplasmin) intravitreal als Alternative an.

In Zukunft muss man sich für diese Fälle eine Fovea-sparing Vitrektomie überlegen, um auf jeden Fall zu vermeiden, dass eine ausgedünnte Zyste durch das Entfernen der Glaskörpertraktion zu einem Makulaforamen wird.

Die Anfärbung des Glaskörpers mittels Triamcinolon führe ich in ausgewählten Fällen durch, etwa bei einem jungen Patienten mit Netzhautabhebung, aber noch teilweise anliegendem Glaskörper.

\section{Anton Haas}

Um eine VMT überhaupt zu behandeln, muss der Visus beeinträchtigt sein (so um $<0,5$ ) und die Netzhaut zystische Veränderungen im OCT aufweisen. Für mich ist die Therapie der Wahl die Vitrektomie, überhaupt bei größeren Adhärenzen $(>1500 \mu \mathrm{m})$. Jetrea ${ }^{\circledR}$ verwenden wir überhaupt nicht, da es zu teuer und für mich dafür zu wenig wirksam ist. Als Alternati- ve wäre bei kleineren Adhärenzen von $<1500 \mu$ m eine intravitreale Einbringung von reinem SF6- oder C3F8Gas zu überlegen. Die Ergebnisse der Studie von Steinle et al. zeigten eine Lösung der VMT in über $80 \%$ der PatientInnen bei Verwendung von C3F8-Gas [23]. Wünschenswert wäre natürlich eine effektivere und auch kostengünstigere enzymatische Vitreolyse. Aber da keine neuen Substanzen derzeit in Phase III-Studien in Erprobung sind, wird wohl die 27 Gauge Vitrektomie die Therapie der Wahl für VMT auch in der näheren Zukunft bleiben. Bezüglich der Frage nach Visualisierung des Glaskörpers habe ich noch keine intraoperative Situation erlebt, wo eine Visualisierung des Glaskörpers notwendig gewesen wäre.

\section{OP-Strategie bei Makulaforamen}

Im Jahr 1991 wurde erstmals die Therapie des Makulaforamens mittels Vitrektomie, Abhebung des Glaskörpers und Gastamponade präsentiert. In dieser Pilotstudie konnte ein Anliegen der am Rand des Foramens elevierten Netzhaut in $58 \%$ der Fälle erreicht werden. Von dieser Gruppe haben wiederum $73 \%$ einen Visusanstieg von zwei oder mehr Zeilen gezeigt [24]. Durch das Entfernen eines vorhandenen Macular Pucker konnte durch die Lösung der Traktionen ein verbessertes Operationsergebnis erzielt werden. Ein zusätzliches Peelen der Membrana limitans interna (inner limiting membrane, ILM) zeigte deutlich bessere Verschlussraten, die bei bis zu $92 \%$ lagen [25]. Erst die Möglichkeit der Anfärbung der transparenten ILM mit Indocyaningrün (ICG) hat zu einer großen Akzeptanz dieses Operationsschrittes geführt [26, 27]. Aufgrund einer möglicher Toxizität von ICG, abhängig von der Applikationsdauer und der Anwendung unter Luft oder Wasser sowie der Konzentration und Art der Verdünnung, sowie die Abhängigkeit der Beleuchtung führte zu zahlreichen experimentellen und klinischen Studien [28]. Als Folge wurden alternative Farbstoffe hinsichtlich ihrer Färbequalität und Toxizität untersucht [29, 30]. Eine weitere Möglichkeit zum Verschluss vor allem großer Makulaforamina wurde 2010 mit der ,inverted ILM Flap“ Technik und 2014 die ILM Transplantation beschrieben [31, 32]. Neben der chirurgischen Intervention hat sich auch die pharmakologische Vitreolyse speziell für Makulaforamen Stadium 2 und 3 mit einen Durchmesser von $<250 \mu$ m etabliert. Im Falle eines persistierenden Makulaforamens stehen verschiedenste Möglichkeiten der Re-Operation zur Verfügung, u. a. wird eine neuerliche Vitrektomie mit einer Silikonöltamponade, die zusätzliche Verwendung von autologem Eigenserum oder auch die Transplantation von einem autologen ILM Flap in der Literatur diskutiert [32-35].

\section{Fragen}

Empfehlen Sie ein ILM-Peeling in allen Stadien des Makulaforamen? 
Mit welchem Farbstoff färben Sie die ILM? Wie applizieren Sie den Farbstoff und wie lange? Sollte zusätzlich eine epiretinale Gliose präsent sein, ändert dies Ihr Vorgehen?

Führen Sie im Rahmen einer ppV bei Makulaforamen beim Wasser-Luftaustausch eine Intervention an der Makula durch? Versuchen Sie die Lochränder des Makulaforamens zu elevieren und dabei das Makulaforamen $\mathrm{zu}$ verkleinern oder sogar zu verschließen? Sehen Sie ein Indikationsspektrum für die „inverted ILM Flap“ Methode?

Angenommen, dass postoperativ die Lochränder nicht mehr eleviert, sondern flach sind, aber das Makulaforamen noch offen ist: Sehen Sie dies als Erfolg oder ist eine Re-Operation indiziert bzw. sinnvoll?

Frage nach der Strategie bei persistierendem Makulaforamen: Welche OP-Technik empfehlen Sie?

\section{Nikolaos Bechrakis}

Das ILM-Peeling empfehle ich bei allen Stadien des Makulaforamen. Ich verwende zur Färbung der ILM Brilliant Blue G, injiziere es nach der Core-Vitrektomie und belasse es, bis der weitere GK bis zur mittleren Peripherie abgetragen worden ist (ca. $60 \mathrm{~s}$ ).

Beim Wasser-Luftaustausch führe ich keine Manipulation der Lochränder durch. Ein Indikationsspektrum für die „inverted ILM Flap“ Methode sehe ich bei Rezidiven und primären $\mathrm{MF} \geq 400 \mu \mathrm{m}$ Durchmesser. Postoperativ sind die Lochränder flach, aber das Makulaforamen ist nicht geschlossen: Dies ist nur als Erfolg zu sehen, wenn der Visus und die Symptome besser geworden sind. Für persistierende Foramen empfehle ich die „inverted ILM Flap“ Methode.

\section{Susanne Binder}

Ich führe bei JEDER Art von Makulaforamen auch ein ILM peeling durch, da damit die Verschlussrate deutlich besser ist.

Ich verwende Vision Blue für $20 \mathrm{~s}$ und färbe manchmal ein 2. Mal um ev. Membranreste aufzufinden.

Den Farbstoff appliziere ich unter offenem Reflux der Infusion, um nicht unter Druck einzuspritzen.

Ich verteile den Farbstoff vorsichtig über den hinteren Pol, verwende nur soviel, wie ich wirklich benötige und teste die Injektionskanüle vorher extraokular, um sicher zu gehen, dass sie nicht verstopft ist.

Wenn ich keinen Flap aus der Membrana limitans interna anlege, um ein großes oder chronisches Foramen zu verschließen, dann berühre ich das Makulaforamen weder mit Instrumenten noch versuche ich durch direktes Absaugen mit der Fluid-Nadel das Loch zu verkleinern.

Ich denke, dass sich die „Flaptechnik“ immer besser etabliert und führe sie in der abgeänderten Technik des temporalen Flaps sehr gerne aus. Indikationen sind im Wesentlichen chronische oder große Makulaforamen, die mit normaler Technik nur eine $70 \%$ Chance auf Verschluss des Foramens haben. Freie
Flaps und Flaps für den Verschluss eines Optic pit werden auch bereits verwendet.

Ein anliegender Lochrand bei noch bestehendem zentralen Defekt kann nur als Teilerfolg gewertet werden. Auch kommt es mit der Zeit häufig noch zu einer Größenzunahme des Defektes mit konsekutiver Seheinbuße.

Bei Re-Operationen ist nur ein freier Flap eine gute Möglichkeit.

Alle anderen Maßnahmen, wie erneute Gaseingabe, Silikontamponade, haben hier wenig Sinn.

Bei persistierendem Makulaforamen habe ich früher Serum bei der Re-Vitrektomie eingesetzt, das aber nur in etwa $50 \%$ der Fälle hilfreich war. Heute setze ich einen freien ILM Flap. Dr. Mamoud aus der Duke University verwendete bei einem myopen, sehr großen Makulaforamen ein freies Netzhauttransplantat erfolgreich.

\section{Anton Haas}

Ich entferne immer die ILM bei allen Stadien der Makulalöcher. Als Farbstoff appliziere ich immer Trypanblau für zirka $20 \mathrm{~s}$, egal ob eine epiretinale Gliose zusätzlich vorhanden ist oder nicht. Den Farbstoff bringe ich über eine Backflush-Kanüle mit Silikonreservoir ein und entferne ihn auch wieder damit, ohne die Kanüle zwischenzeitlich aus dem Auge zu nehmen.

Beim Wasser-Luftaustausch halte ich am Ende eine Backflush-Kanüle knapp über das Makulaloch, um Flüssigkeit aus dem Loch zu saugen. Dabei legen sich die Lochränder meist an und das Loch verkleinert sich. Einen kompletten Verschluss versuche ich nie zu erreichen.

Einen „inverted ILM Flap“ mache ich immer bei Makulalöchern bei höherer Myopie.

Wenn nach dem Ersteingriff das Loch nicht verschlossen ist, egal ob mit elevierten oder flachen Lochrändern, so führe ich eine Revitrektomie mit Silikonöl durch. Persistierende Makulaforamina auch mit elevierten Rändern können damit meist mit Visusgewinn verschlossen werden.

\section{OP Strategie bei der Ablatio retinae}

Die rhegmatogene Ablatio retinae führt ohne Therapie zu einem zunehmenden Sehverlust. Eine verzögerte Therapie ist mit einer limitierten Sehverbesserung assoziiert [36]. Seit 1929 ist die chirurgische Intervention mit Behandlung des verursachenden Netzhautdefektes beschrieben [37]. Verschiedenste Operationsmethoden haben sich im Verlauf etabliert, relevant sind heute die Plombenoperation, die pneumatische Retinopexie und die ppV [38]. Die pneumatische Retinopexie ist in den USA immer noch populär, in Europa wird diese Methode zu Gunsten der Plombenoperation und ppV deutlich seltener durchgeführt. Gründe sind die erhöhte Rate an proliferativer Vitreoretinopathie (PVR) und das postoperativ erhöhte Risiko für neue Netzhautdefekte. Die Plombenoperation 
hat sich seit der Einführung dieser Technik deutlich verändert [39]. Während früher die Plombenoperation vielfach mit zusätzlichen Behandlungen wie Punktion und auch mit einer zusätzlichen intraokulären Gastamponade kombiniert wurde, definiert die minimale Buckelchirurgie die alleinige lokale Plombenapplikation mit Retinopexie im Bereich der Netzhautdefekte und ist somit ein rein extraokulärer Eingriff. Entsprechend der SPR-Study hat sich für die Gruppe der phaken Augen ein besserer postoperativer Visus für die Gruppe der Plombenoperation im Vergleich zur Gruppe der ppV gezeigt [40]. In der SPR-Study wurden nur Fälle mit mittlerem Schweregrad eingeschlossen. Der Anteil an ppV im Vergleich zur Plombenoperation zur Behandlung der Ablatio retinae hat kontinuierlich zugenommen. Gründe sind ein besseres, angenehmeres OP-Setting bei der ppV im Vergleich zur Plombenchirurgie. Die in den letzten Jahren deutlich verbesserte und somit erleichterte Durchführung der Vitrektomie und die Tatsache, dass die $\mathrm{ppV}$ auch für andere vitreoretinale Erkrankungen etabliert ist, führt zu mehr Operationserfahrung für die ppV. Das Erlernen der Buckelchirugie wird von vielen Chirurgen als schwer angesehen, und aufgrund der geringeren Operationszahlen stehen weniger erfahrene Kollegen für die Lehre zur Verfügung [41, 42].

\section{Fragen}

Welche OP Technik wenden Sie zur Therapie der Ablatio retinae und wovon ist dies abhängig? Wie lange kann mit der Ablatio-Operation zugewartet werden?

Angenommen es besteht eine inferiore massive PVR mit Sternfalten in der mittleren Peripherie. Die inferiore Netzhaut lässt sich nach Peelen der Membranen mit Perfluorocarbon nicht anlegen. Welche Strategie wählen Sie?

Welche Lichtquelle verwenden Sie?

Sehen Sie eine Indikation für schwere Silikonöle?

Wie führen Sie die präoperative und die postoperative Lagerung der Patienten mit Ablatio retinae durch?

\section{Nikolaos Bechrakis}

Bei Pseudophakie führe ich eine primäre 25 Gauge PPV, bei phaken <ca. 45 Jahren und klarer Lochsituation: Plombe, bei Kindern fast immer Plombe.

Wie lange kann mit der Operation zugewartet werden? Bei drohender Ablösung und gerade abgelöster Fovea führe ich die Operation am gleichen Tag, bei länger bestehender abgelöster Makula in den nächsten 1-3 Tagen durch und bei einer chronisch asymptomatischen Ablatio vergebe ich einen elektiven Termin.

In dem beschriebenen Fall mit inferiorer PVR führe ich eine ausgedehnte Retinektomie mit Silikonöl Tamponade durch.

Als Lichtquelle verwende ich bei der Makulachirurgie ein handheld-spot, bei Ablatio/bimanuellen Manipulationen (z. B. PDR): Chandelier (entweder 25 oder $27 \mathrm{~g}$ ).
Eine Indikation für schwere Silikonöle sehe ich nur bei Patienten, die nicht auf dem Bauch liegen können, dies aber tun müssen, sonst nicht.

Bezüglich der Lagerung empfehle ich präoperativ Bettruhe und Loch nach unten lagern und postoperativ am gleichen OP-Tag Makula nach oben lagern (unabhängig von der Lochlage), ab dem 1. postoperativen Tag und nachdem die Makula sicher anliegt, Loch nach oben lagern.

\section{Susanne Binder}

Zu $90 \%$ primäre Vitrektomie mit oder ohne Kataraktoperation und IOL-Implantation.

Bei $10 \%$ ist immer noch ein Buckel indiziert, allerdings setzen wir dabei ein Chandelierlicht ein und verwenden das Biom zur Visualisierung, also eine mikrochirurgische Buckelchirurgie.

Ist die Makula bedroht, so wenig wie technisch möglich. Priorität hat aber, dass ein erfahrener Netzhautchirurg zur Verfügung steht. Ist die Makula bereits abgehoben, kann man 2, maximal 3 Tage warten.

Wenn sich die Netzhaut inferior nicht entfalten lässt, dann führe ich eine großzügige Retinektomie durch. Handelt es sich um ein einziges bzw. letztes Auge, dann überlege ich ebenfalls eine unterstützende Cerclage, da mir diese Kombination eine größere Sicherheit gibt, die Silikonöltamponade auch wieder komplikationsfrei entfernen zu können.

Für schwere Fälle Chandelierlichter, um besser mit beiden Händen präparieren zu können.

Schwere Silikonöle verwende ich schon lange nicht mehr, da sie inflammatorisch sind und schwere Komplikationen machen können. Durch unsere verbesserte Vitrektomietechnik ist der Einsatz auch kaum mehr nötig.

Präoperativ wird nur einem Patienten mit Makulabedrohung geraten das Bett zu hüten.

Postoperativ wird primär für die ersten Stunden am Bauch gelagert, dann nach der entsprechenden Seite, die der Pathologie gegenüber liegt. Am nächsten Tag wird die Lagerungsempfehlung meist schon aufgehoben.

\section{Anton Haas}

Meistens führe ich bei Ablationes eine 23 Gauge Vitrektomie mit C3F8 durch, bei PVR-Reaktionen, vor allem in der unteren Netzhauthälfte, verwende ich Silikonöl. Nur bei jungen Patienten und bei Netzhautlöchern zwischen 4 und 8 Uhr überlege ich eine Plombe.

Der Zeitpunkt der Operation hängt prinzipiell davon ab, ob die Makula abgehoben ist oder nicht. Bei anliegender Makula setze ich die chirurgische Sanierung innerhalb von $24 \mathrm{~h}$, bei abgehobener kann durchaus 2-3 Tage abgewartet werden, aber aus psychologischer Sicht operiere ich so schnell wie möglich.

Bei dieser Situation mache ich eine inferiore Retinotomie und Retinektomie mit Silikonöl.

Ich verwende eine Xenon-Lichtquelle (Photon II $\left.{ }^{\circledR}\right)$. 
Eine Zeit lang habe ich schwere Silikonöle mit nicht überzeugendem Erfolg verwendet. Ich habe aber wieder damit aufgehört.

Die präoperative Lagerung hängt von der Lage des Netzhautdefektes ab. Bei Löchern und Abhebung oben verordne ich flache Liegeposition, bei Löchern und Abhebung unten eine Position mit eher aufrechtem Oberkörper. Unmittelbar postoperativ empfehle ich eine Gesichtslage über die Nacht, um eine Rotation der Makula zu verhindern, danach eine Lagerung abhängig vom Netzhautdefekt.

\section{Die Zukunft der Vitrektomie}

\section{Fragen}

Welche Größe der Trokare wird sich etablieren? Welche neuen Trends können wir erwarten? Wird das Indikationsspektrum weiter zunehmen oder müssen wir damit rechnen, dass alternative Behandlungsmethoden die Vitrektomie ersetzen können?

\section{Nikolaos Bechrakis}

Alle 3 Größen der Trokare werden sich etablieren. Wir werden in Zukunft mit einer besseren Visualisierung und mit besseren Schneide-Mechanik rechnen können. Weder wird das Indikationsspektrum zunehmen oder die Vitrektomie ersetzt werden, dies ist aber sehr spekulativ.

\section{Susanne Binder}

Denke, dass wir bei 25-27 Gauge lange Zeit bleiben.

Die heads-up Chirurgie wird sich in der Hinterabschnittschirurgie etablieren, Diagnostik wie OCT etc. wird integriert werden.

Indikationen werden zunehmen, ob es allerdings im Bereich der „Floatervitrektomie“ sein wird, ist schwer vorherzusagen.

\section{Anton Haas}

Meiner Meinung wird sich die Trokargröße bei 25 Gauge einpendeln, da sich damit auch hochvisköseres Silikonöl einbringen lässt. Die 27 Gauge Vitrektomie wird sicher bei der Makulachirurgie ihre Berechtigung haben.

Die neueren Trends gehen sicher in Richtung präzisere und effizientere Vitrektomiegeräte und -instrumente, und wir werden auch prüfen, ob die neue Ultraschalltechnik gegenüber der herkömmlichen pneumatischen Vitrektomie einen Vorteil bieten wird. Eingang in die Vitrektomie werden auch in das Mikroskop integrierte bildgebende Verfahren finden, die ein präziseres und sicheres Arbeiten ermöglichen. Es werden auch neue pharmakologisch wirksame Substanzen auf den Markt kommen, die eine Vitrektomie unterstützen und erleichtern, und damit bessere Ergebnisse liefern.
In näherer Zukunft sehe ich aber keine wesentliche Zunahme des Indikationsspektrums und auch keine Alternativen zur Vitrektomie.

Open access funding provided by University of Innsbruck and Medical University of Innsbruck.

Interessenkonflikt S. Mennel, N.E. Bechrakis, S. Binder und A. Haas geben an, dass kein Interessenkonflikt besteht.

Open Access This article is distributed under the terms of the Creative Commons Attribution 4.0 International License (http://creativecommons.org/licenses/by/4.0/), which permits unrestricted use, distribution, and reproduction in any medium, provided you give appropriate credit to the original author(s) and the source, provide a link to the Creative Commons license, and indicate if changes were made.

\section{Literatur}

1. Machemer R, Buettner H, Norton EW, Parel JM. Vitrectomy: a pars plana approach. Trans Am Acad Ophthalmol Otolaryngol. 1971;75:813-20.

2. BüttnerH, Machemer R, ParelJM.Vitrectomy. 1.Instrumentarium and operative technic. Ber Zusammenkunft Dtsch OphthalmolGes. 1972;71:424-8.

3. Machemer R. The development of pars plana vitrectomy: a personal account. Graefes Arch Clin Exp Ophthalmol. 1995;233:453-68.

4. Charles S. The history of vitrectomy: innovation and evolution. Retina Today. 2008;5:27-9.

5. de Oliveira PR, Berger AR, Chow DR. Vitreoretinal instruments: vitrectomy cutters, endoillumination and wideangle viewing systems. Int J Retin Vitreous. 2016;2:28.

6. Chang S, Lincoff H, Zimmerman NJ, Fuchs W. Giant retinal tears. Surgical techniques and results using perfluorocarbon liquids. Arch Ophthalmol. 1989;107:761-6.

7. Chen JC. Sutureless pars plana vitrectomy through selfsealing sclerotomies. Arch Ophthalmol. 1996;114:1273-5.

8. Schmidt J, Nietgen GW, Brieden S. Self-sealing, sutureless sclerotomy in pars plana vitrectomy. Klin Monbl Augenheilkd. 1999;215:247-51.

9. Rauber M, Mester U. Incidence and prophylaxis of retinal detachment following pars plana vitrectomy. Ophthalmologe. 2006;103:673-6.

10. Schmidt JC, Meyer CH, Mennel S. Pars-plana vitrectomy with anterior chamber infusion via a paracentesis in pseudophakic eyes. Ophthalmologe. 2007;104:222-5.

11. Fujii GY, De Juan EJr, Humayun MS, Pieramici DJ, ChangTS, Awh C, Ng E, Barnes A, Wu SL, Sommerville DN. A new 25gauge instrument system for transconjunctival sutureless vitrectomy surgery. Ophthalmology. 2002;109:1807-12.

12. Eckardt C. Transconjunctival sutureless 23 -gauge vitrectomy. Retina (Philadelphia, Pa). 2005;25:208-11.

13. Oshima Y, Wakabayashi T, Sato T, Ohji M, Tano Y. 27gauge instrument system for transconjunctival sutureless microincision vitrectomy surgery. Ophthalmology. 2010;117:93-102.

14. Schmidt JC, Mennel S, Rodrigues EB, Meyer CH. Enhanced visualisation of the vitreous during bimanual vitreous shaving by trans-scleral illumination. Br J Ophthalmol. 2010;94:140.

15. Oshima Y, Awh CC, Tano Y. Self-retaining 27-gauge transconjunctival chandelier endoillumination for panoramic viewing during vitreous surgery. Am J Ophthalmol. 2007;143:166-7. 
16. Stalmans P, Benz MS, Gandorfer A, Kampik A, Girach A, Pakola S, Haller JA, MIVI-TRUST Study Group.. Enzymatic vitreolysis with ocriplasmin for vitreomacular traction and macular holes. NEngl J Med. 2012;367:606-15.

17. Bertelmann T, Wachtlin J, Mennel S, Koss MJ, Maier MM, Schumann RG, Kazerounian S, Daniel H, Schmitz-Valckenberg S, EXPORT study group. The predictability of ocriplasmin treatment effects: Is there consensus among retinal experts? Results from the EXPORT study. Graefes Arch Clin Exp Ophthalmol. 2017;255(7):1359-67. doi:10. 1007/s00417-017-3657-2.

18. García-Layana A, García-Arumí J, Ruiz-Moreno JM, AriasBarquet L, Cabrera-López F, Figueroa MS. A review of current management of vitreomacular traction and macular hole.JOphthalmol.2015;2015:809640.

19. MennelS. EnzymatischeVitreolyse-von derklinischenStudie zur praktischen Anwendung. Spektrum Augenheilkd. 2014;28:59-60.

20. Ochoa-Contreras D, Delsol-Coronado L, Buitrago ME, Velasco-Barona C, Quiroz-Mercado H. Induced posterior vitreous detachment by intravitreal sulfur hexafluoride (SF6) injection in patients with nonproliferative diabetic retinopathy. Acta Ophthalmol Scand.2000;78:687-8.

21. Claus MG, Feron E, Veckeneer M. Pneumatic release of focal vitreomacular traction. Eye (Lond). 2017;31:411-6.

22. Chan CK, Crosson JN, Mein CE, Daher N. Pneumatic vitreolysis for relief of vitreomacular traction. Retina (Philadelphia,Pa).2017; doi:10.1097/iae.0000000000001448.

23. Steinle NC, Dhoot DS, Quezada Ruiz C, Castellarin AA, Pieramici DJ, See RF, Couvillion SC, Nasir MA, Avery RL. Treatment of vitreomacular traction with intravitreal perfluoropropane (C3F8) injection. Retina (Philadelphia, Pa). 2017;374:643-50.

24. Kelly NE, Wendel RT. Vitreous surgery for idiopathic macular holes. Results of a pilot study. Arch Ophthalmol. 1991;109:654-9.

25. Eckardt C, Eckardt U, Groos S, Luciano L, Reale E. Removal of the internal limiting membrane in macular holes. Clinical and morphological findings. Ophthalmologe. 1997;94:545-51.

26. Kadonosono K, Itoh N, Uchio E, Nakamura S, Ohno S. Staining of internal limiting membrane in macular hole surgery. Arch Ophthalmol. 2000;118:1116-8.

27. Meyer CH, Schmidt JC, Mennel S, Göddeke E, Rübe $\mathrm{K}$, Rodrigues EB, Kroll P. Anatomical and functional results after macular hole surgery. Klin Monbl Augenheilkd. 2008;225:220-6.

28. Mennel S, Thumann G, Peter S, Meyer CH, Kroll P. Influence of vital dyes on the function of the outer blood-retinal barrier in vitro. Klin Monbl Augenheilkd. 2006;223:568-76.

29. Mennel S, Meyer CH, Tietjen A, Rodrigues EB, Schmidt JC. Patent blue: a novel vital dye in vitreoretinal surgery. Ophthalmologica. 2006;220:190-3.
30. Rodrigues EB, Meyer CH, Mennel S, Farah ME. Mechanisms of intravitreal toxicity of indocyanine green dye: implications for chromovitrectomy. Retina (Philadelphia, Pa). 2007;27:958-70.

31. Michalewska Z, Michalewski J, Adelman RA, Nawrocki J. Inverted internal limiting membrane flap technique for large macular holes. Ophthalmology. 2010;117:2018-25.

32. Morizane Y, Shiraga F, Kimura S, Hosokawa M, Shiode Y, Kawata T, Hosogi M, Shirakata Y, Okanouchi T. Autologous transplantation of the internal limiting membrane for refractory macular holes. Am J Ophthalmol. 2014;157:861-9.

33. Lai JC, Stinnett SS, McCuen BW. Comparison of silicone oil versus gas tamponade in the treatment of idiopathic fullthickness macular hole. Ophthalmology. 2003;110:1170-4.

34. Ozdek S, Baskaran P, Karabas L, Neves PP. A modified perfluoro-n-octane-assisted autologous internal limiting membrane transplant for failed macular hole reintervention: a case series. Ophthalmic Surg Lasers Imaging Retina. 2017;48:416-20.

35. Dimopoulos S, William A, Voykov B, Ziemssen F, BartzSchmidt KU, Spitzer MS. Anatomical and visual outcomes of autologous thrombocyte serum concentrate in the treatment of persistent full-thickness idiopathic macular hole after ILM peeling with brilliant blue $\mathrm{G}$ and membrane blue dual. Acta Ophthalmol. 2016;19 doi:10.1111/aos.12971.

36. Liu F, Meyer CH, Mennel S, Hoerle S, Kroll P. Visual recovery after scleral buckling surgery in macula-off rhegmatogenous retinal detachment. Ophthalmologica. 2006;220:174-80.

37. Gonin J. Le traitement operatoire du decollement retinien. Conference auxjournees medicales de Bruxelles. BruxellesMed. 1930;23:17.

38. Kreissig I. Surgical techniques for repair of primary retinal detachment: part I. Review of their development during the last 80 years. Folia Med (Plovdiv). 2009;51:5-11.

39. Kreissig I. Treatment of primary retinal detachment. Minimal extraocular or intraocular? Ophthalmologe. 2002;99:474-84.

40. Heimann H, Bartz-Schmidt KU, Bornfeld N, et al. Scleral buckling versus primary vitrectomy in rhegmatogenous retinal detachment: a prospective randomized multicenter clinical study. Ophthalmology. 2007;114:2142-54.

41. Georgopoulos M, Sacu S, Eibenberger K, Blum R, Reza S, Schmidt-Erfurth U. Entwicklung der Operationstechniken bei Netzhautablösung. (Development of surgical approach to rhegmatogenous retinal detachment). Spektrum Augenheilkd.2012;26:92.

42. Mennel S, Thaler M, Reichart S. Vitrektomie oder Plombenchirurgie zur Behandlung der rhegmatogenen Ablatio retinae - Vitrectomy or buckling surgery to treat rhegmatogenous retinal detachment. Spektrum Augenheilkd. 2013;27:100. 